\title{
Implementation of an Engineering-Based Retention Center and its Impact on Student Success
}

\section{Jaclyn Marie Esqueda, University of Wisconsin, Platteville}

Jaclyn is a Special Programs Manager in the College of Engineering, Mathematics and Science Student Success Programs department at the University of Wisconsin-Platteville. She received her Master of Education degree in Counselor Education-Higher Education Student Services from Georgia Southern University. She is strongly committed to helping all students find a meaningful and life-changing educational experience in an inclusive campus environment.

\section{Dr. Christina Curras, University of Wisconsin, Platteville}

Dr. Curras earned her PhD in Geotechnical Engineering at the University of California at Davis. She has been a faculty member at the University of Wisconsin-Platteville since 2000, and she currently is serving as the Chair of the Department of Civil and Environmental Engineering and as the Assistant Dean for Student Services for the College of Engineering, Mathematics, and Science. 


\section{Implementation of an Engineering-Based Retention Center and its Impact on Student Success}

\section{Introduction}

Undergraduate student retention and graduation rates are, and have been for many years, frequently discussed topics within the higher education community. The May 2015 Condition of Education report from the National Center for Education Statistics puts the current first-year retention rate for four-year institutions at $80 \%$ and the six-year graduation rate for four-year institutions at $59 \%{ }^{4}$. With the national attention and focus on these numbers and their implications of a university's academic health and student success, many college and university administrators are developing stronger strategic priorities and goals for student persistence.

In addition, there has been significant national attention on STEM education and ensuring that the country has enough qualified individuals to meet workforce needs ${ }^{1}$. A 2013 Department of Education report states that, "a total of $48 \%$ of bachelor's degree students . . . who entered STEM fields between 2003 and 2009 had left these fields by spring 2009" (p. 14) ${ }^{3}$. This indicates that almost half of students who have an interest in pursuing a STEM degree either change to a non-STEM major, or drop out of school altogether ${ }^{3}$. This, too, has led to a closer examination of and focus on college retention rates.

For those of us who are "on the front lines," so to speak - those of us who have daily interactions with students and who take a direct and active helping role in their educational pursuits - that often means finding creative new ways to tackle this entrenched and many-headed monster. The reasons for student attrition are complex and varied, and therefore it stands to reason that the solution (or solutions, more likely) must also be multi-pronged. On the ground level, it is about understanding students' needs and attempting to partner with them to meet those needs in pragmatic ways. It is not usually about dramatic, sweeping changes. More often, it is about trying this new advising technique, adopting that new teaching method, or making this slight modification to an existing program. And slowly, collectively, these efforts begin to move the needle.

One such promising potential solution was developed at the University of Wisconsin-Platteville (UW-Platteville). UW-Platteville is a medium-sized, primarily undergraduate, public institution with a total undergraduate enrollment of approximately 8,000. Many of the university's STEM degree programs, including seven engineering programs, computer science, and chemistry, are housed within the College of Engineering, Mathematics and Science (EMS), which has an enrollment of approximately 2,700 students.

In 2013, with a desire to improve retention and persistence of students in the college, an interdisciplinary team of faculty and staff obtained a National Science Foundation STEM Talent 
Expansion Program grant. The primary purpose of the grant is to increase undergraduate student engagement and to plan, market, and advance programs that support recruitment and retention efforts in STEM with special efforts toward women and underrepresented student populations.

As part of achieving this purpose, a study and resource space was created within the College of EMS. Two commonly discussed reasons for students leaving STEM fields are academic difficulty and failure to integrate effectively into the campus community or feelings of isolation/not fitting $\mathrm{in}^{2}$. A space of this nature, in theory, would address both of these concerns it would provide academic support and also provide a welcoming atmosphere that allows for natural interaction with like-minded peers within the larger campus community. Prior to the creation of this center, many informal study alcoves and spaces existed within classroom buildings on the campus, but none that were staffed or provided consistent access to resources.

\section{Implementation}

A classroom in one of the two engineering academic buildings was identified and approved by the university administration for the center's location. An interactive forum was held in the spring of 2013 in the classroom that would be the future location of the center. The purpose of the forum was to gather input from students before constructing the space. All students in the College of EMS were invited to attend. The forum consisted of several interactive stations, including: design boards where students could vote on their preferred color scheme; white boards where students could suggest and vote for their favorite potential center names; Legos where students could build and photograph their ideal layout for the room; sample furniture that students could try out; and a survey with questions about preferred study hours, lighting, seating, technology, staff, and services. Door prizes and snacks were also included as part of the event.

The room, named CenterPOINT (Center for Projects, Opportunities, Instruction, Networking, and Teamwork), was remodeled over the summer of 2013 incorporating the feedback received at the forum. Upon its opening, CenterPOINT included such features as: a full-time academic advisor/center manager; a front desk staffed by student assistants; free drop-in peer tutoring hours; tables on wheels that could be easily moved into different configurations; five computer stations; items available for checkout such as phone chargers, headphones, and calculators; a kitchenette space with refrigerator, microwave, and sink; wall-mounted and mobile white boards; soft seating; and cubby storage for books and personal belongings. The space comfortably seats approximately 34 people. A floorplan for the initial layout of the room can be seen in Appendix A.

During the summer of 2013, student staff positions were advertised through the university's student employment job board and applications were solicited. The center manager and a student assistant conducted a screening and interview process and selected a team of eight student staff. A training retreat was held with the full team and included review of job duties and work 
policies, discussion of providing excellent service to CenterPOINT users, and teambuilding activities. Each staff member also participated in one-on-one, on-the-job training with the center manager.

In order to promote the new space, a grand opening event was held at the start of the fall 2013 semester. University administrators and all students, staff, and faculty in the College of EMS were invited to attend and learn about the space and its services. Email communications, a campus press release, and flyers were used to promote the open house and the new space. The space is also advertised continuously through a website and Facebook page.

Initially, CenterPOINT was open and staffed seven days per week for a total of 81 hours per week. Hours were Monday through Thursday from 8:00 am to 10:00 pm, Friday from 8:00 am to 5:00 pm, Saturday from 1:00 to 8:00 pm, and Sunday from 1:00 to 10:00 pm. After analyzing usage during the first year, scheduled Saturday hours were eliminated and students instead use a reservation request process if they would like to use the space on Saturdays. Hours on all other days remain the same.

Improvements are continuously made as students voice their suggestions. In semesters after the space opened, several components were added including: availability of engineering paper; a single-serve coffee brewing machine; a collaboration station where multiple students can plug in laptops and display their screen on a large monitor; laptops available for check out; dual monitors on the computer stations; and rotating art pieces from the campus's permanent gallery collection.

\section{Assessment Methods}

Several different assessment tools have been used to measure the success of the space and its services, including data analysis on user demographics, surveys, and focus groups.

When students enter the space, they are asked by staff at the front desk to swipe their student ID through a card reader device. If they do not have their ID card with them or prefer not to swipe, they are given the option to sign in manually instead, or to decline to sign in at all. The card reader stores a 16-digit unique identifier, date, and time. This ensures that the student users' information is kept confidential. The 16-digit numbers are later converted into demographic data by the center manager. From this data, number of visits and unique visitors can be determined. Additional data can also be obtained regarding student users' academic achievement and retention.

As a second method of tracking space usage, desk staff manually tally the usage of the space once each hour. They look for which components of the room are being used at that point in time 
and record it in a spreadsheet. From this data, percent occupancy as well as most popular items can be determined.

Because the space is intended to serve students, their feedback is essential. A 10-question electronic survey is issued via email to all student users at the end of each academic year. Questions that measure student users' decision to return to the center, feelings of comfort and fitting in, and impact on academic success are included, among others. The complete survey appears in Appendix B.

In order to drill down and obtain additional information about student users' experience of the space, focus groups were held during the spring 2015 semester. External evaluators were hired to design and conduct the focus groups to minimize bias. Four total groups were held - two control groups of non-CenterPOINT users and two groups of CenterPOINT users. The same questions were asked in each of the four groups.

\section{Outcomes}

Visits and Visitor Demographics

Table 1 shows usage of the space in CenterPOINTS's first two complete academic years. These unique user numbers represent approximately $30 \%$ of College of EMS students in 2013-14, and approximately $35 \%$ in 2014-15.

Table 1: CenterPOINT Visitors

\begin{tabular}{|l|l|c|c|}
\hline Year & Visits & Unique Visitors & Average Visits per Visitor \\
\hline Academic Year 2013-14 & 12,932 & 816 & 16 \\
\hline Academic Year 2014-15 & 13,956 & 933 & 15 \\
\hline
\end{tabular}

In 2013-14 overall, 29.4\% of visitors were female (Table 2). This is significantly higher than the percentage of women enrolled in the College of EMS overall, which was $14.3 \%$ in the 2013-14 academic year.

Table 2: CenterPOINT Visitors by Gender, 2013-14

\begin{tabular}{|l|l|l|}
\hline & Male & Female \\
\hline CenterPOINT 2013-2014 & $70.6 \%$ & $29.4 \%$ \\
\hline College of EMS 2013-2014 & $85.6 \%$ & $14.3 \%$ \\
\hline
\end{tabular}

In 2013-14 overall, $11.5 \%$ of visitors were underrepresented minority students as compared to 8.5\% underrepresented minority students within the College of EMS as a whole, shown in Table 3. 
Table 3: CenterPOINT Visitors by Race/Ethnicity, 2013-14

\begin{tabular}{|l|c|c|c|}
\hline & Underrepresented & White & Unknown \\
\hline CenterPOINT Fall 2013 & $10.3 \%$ & $88.8 \%$ & $0.2 \%$ \\
\hline College of EMS Fall 2013 & $7.7 \%$ & $92.0 \%$ & $0.3 \%$ \\
\hline CenterPOINT Spring 2014 & $12.7 \%$ & $87.2 \%$ & $1.2 \%$ \\
\hline College of EMS Spring 2014 & $8.3 \%$ & $91.5 \%$ & $0.3 \%$ \\
\hline
\end{tabular}

By class standing (Table 4), the fall semester showed some uneven distribution; notably, freshmen were underrepresented and seniors were overrepresented. This had balanced out by spring semester, where spring users were distributed more proportionately to the college's breakdown.

Table 4: CenterPOINT Visitors by Class Standing, 2013-14

\begin{tabular}{|l|l|l|l|l|}
\hline & Freshman & Sophomore & Junior & Senior \\
\hline CenterPoint Fall 2013 & $12.5 \%$ & $17.5 \%$ & $24.5 \%$ & $45.5 \%$ \\
\hline College Fall 2013 & $33.5 \%$ & $20.5 \%$ & $19.7 \%$ & $26.3 \%$ \\
\hline CenterPoint Spring 2014 & $21.7 \%$ & $21.9 \%$ & $23.7 \%$ & $32.4 \%$ \\
\hline College Spring 2014 & $25.0 \%$ & $20.6 \%$ & $21.2 \%$ & $33.2 \%$ \\
\hline
\end{tabular}

In terms of major, Table 5 shows that Civil Engineering is overrepresented while some other disciplines, such as Electrical Engineering, are underrepresented. This can be explained primarily because of the location of the space - it is in the building that houses the civil, environmental, general, and mechanical engineering departments whereas Electrical Engineering is in a building a significant distance away.

Table 5: CenterPOINT Visitors by Major, 2013-14

\begin{tabular}{|l|c|c|}
\hline Major & CenterPOINT & College of EMS \\
\hline Civil Engineering & $26 \%$ & $14 \%$ \\
\hline Electrical Engineering & $6 \%$ & $12 \%$ \\
\hline Environmental Engineering & $7 \%$ & $4 \%$ \\
\hline Engineering Physics & $5 \%$ & $5 \%$ \\
\hline General Engineering & $3 \%$ & $7 \%$ \\
\hline Industrial Engineering & $7 \%$ & $5 \%$ \\
\hline Mechanical Engineering & $29 \%$ & $34 \%$ \\
\hline Software Engineering & $2 \%$ & $5 \%$ \\
\hline Other College of EMS Majors & $8 \%$ & $15 \%$ \\
\hline Majors Outside of EMS & $9 \%$ & $0 \%$ \\
\hline
\end{tabular}

\section{Retention}

Students who use CenterPOINT retain to their next year of education at a rate of $86.6 \%$, as compared to $73.7 \%$ of their peers who do not utilize the space. This difference is significant with a p-value of 0.000 , using a two proportions hypothesis test. 


\section{Grade Point Averages}

Students who utilize CenterPOINT have cumulative GPA's that average .24 higher than their peers who do not use the space. CenterPOINT users' term GPA's are .27 higher on average. Both results are statistically significant at a .01 significance level.

Table 6: CenterPOINT Visitors' Grade Point Averages

\begin{tabular}{|l|l|l|}
\hline & $\begin{array}{l}\text { Cumulative } \\
\text { GPA }\end{array}$ & Term GPA \\
\hline CenterPOINT Users & 3.06 & 2.93 \\
\hline CenterPOINT Nonusers & 2.82 & 2.66 \\
\hline
\end{tabular}

\section{Space Usage}

In terms of type of study, $61.5 \%$ of visitors utilize the space for individual study, and $38.5 \%$ of visitors use it for group study. The most commonly used features of the space are the study tables (47\% of overall space usage), computer stations (34\% of overall space usage), collaboration station large monitor with laptop hookups (9.5\% of overall space usage), and soft seating tablet arm chairs ( $8 \%$ of overall space usage).

Peak usage of CenterPOINT typically occurs during the 10:00 am hour, with a consistent average of $60 \%$ capacity achieved during that time.

\section{Survey Results}

To date, the CenterPOINT user survey has been issued three times. In the first year of operation, the survey was issued at the end of each semester. After collecting this initial year data, the survey process was revised and it is now issued annually at the end of each academic year. Results consistently indicate high levels of user satisfaction. A summary of results from key survey questions is highlighted in Tables 7 and 8 below.

Table 7: Top Factors that Influenced Users to Return to CenterPOINT

\begin{tabular}{|l|c|}
\hline Factor & $\begin{array}{l}\% \text { of Users Influenced } \\
\text { by this Factor }\end{array}$ \\
\hline Convenience of location & $86 \%$ \\
\hline Atmosphere of space & $77 \%$ \\
\hline Availability of resources & $68 \%$ \\
\hline Staff & $51 \%$ \\
\hline
\end{tabular}


Table 8: CenterPOINT User Survey Results - Key Questions

\begin{tabular}{|l|r|r|r|}
\hline & \% Who Selected "Agree" or "Strongly Agree" \\
\hline Question & Fall 2013 & Spring 2014 & Fall 2014 \\
\hline I feel I fit in at the CenterPOINT. & $83.6 \%$ & $86.3 \%$ & $82.7 \%$ \\
\hline $\begin{array}{l}\text { CenterPOINT provides a welcoming } \\
\text { environment. }\end{array}$ & $88.8 \%$ & $89.0 \%$ & $90.8 \%$ \\
\hline I feel comfortable while at the CenterPOINT. & $87.9 \%$ & $90.4 \%$ & $91.8 \%$ \\
\hline
\end{tabular}

Students are also given the opportunity to provide additional comments on the survey. A few representative comments are included here:

- "CP has been my go-to study spot this semester and my one area where I can always organize my thoughts. I run on a VERY busy schedule ... and this room has given me a place to relax and be productive."

- "The CenterPOINT provides a comfortable environment that enhances my productivity while studying."

- "I know that there are people there usually that are in my classes for me to study with or ask a quick question which helps me complete homework more efficiently."

- "CenterPOINT is always comfortable, and there are several people who can help with classes, both friends and [peer-assisted learners]. It is much quieter than the atrium and provides much better areas to study than a computer lab."

- "I used CenterPOINT to work with fellow students on projects and assignments for class that helped me overall do better in the class."

\section{Focus Group Results}

After the focus group conversations were transcripted and analyzed, several themes emerged. When asked about specific services or spaces on campus that they find beneficial and why, students mentioned that access to resources like computers and whiteboards and the environment of the space is what caused them to prefer certain places over others. This is consistent with what the survey results demonstrated.

A second theme that arose was related to crowding and noise level in CenterPOINT. Several students commented that they often could not find a spot, or that because of others working in groups, the volume in the space made it difficult for them to focus on their own work. This provides support for the need for more spaces like CenterPOINT on campus, and also for dedicated quiet study areas in academic classroom buildings.

Several students discussed awareness of resources and spaces and mentioned that they were unaware of what is available. Suggestions were made for how to better market and promote CenterPOINT to potential student users. 


\section{Qualitative Outcomes}

There are several other noteworthy outcomes of the process of implementing CenterPOINT. Through development of the space and its services, strong partnerships have been developed with the campus's Math Learning Center, University Tutoring Services, and Office of Multicultural Student Affairs, helping to create a more inclusive space with expanded services for students. These expanded services and important partnerships are important steps toward the process of institutionalizing the space.

Many of the policies and training sessions developed for CenterPOINT staff have been modified and adopted for use within other departments on the campus, demonstrating that others find value in the structure that these elements provide.

Recently, during a minor remodel of a portion of the classroom building where CenterPOINT is housed, administration asked for input from CenterPOINT staff regarding the best use of a small space that had been created by the division of a classroom. Based on direct feedback from the survey and focus group results, a recommendation was made that the space be used for a designated quiet study area. This was implemented in the fall 2015 semester and put under the purview of CenterPOINT.

\section{Challenges and Limitations}

There are some factors in the implementation of the CenterPOINT and some limitations to these results which may make the overall process less generalizable to other institutions.

If an appropriate space or location does not already exist for a center of this nature, it may be cost prohibitive for some institutions to create one. Because CenterPOINT is part of a grant initiative, portions of the startup cost, such as the staffing and some supplies, were covered by external dollars. Also, due to administrative support and collaborative partnerships, parts of the remodel expenses were covered with indirect money that resulted from the receipt of the grant. Even with initial costs partially externally funded, the institutionalization and maintenance of the space and its staff and resources can lead to difficult budget decisions.

Another limitation or caution of applying these results more generally is related to the scope of the project. The objectives of the STEP grant at UW-Platteville are many, and CenterPOINT has been one piece of an overall larger effort to improve retention in the College of EMS. While CenterPOINT has proven to be a very effective resource, it is important to note that the implementation of the center did not occur in isolation and many other factors likely contribute to its success. An existing department on campus, called Engineering, Mathematics and Science Student Success Programs, is the parent department of CenterPOINT and provides important support. 


\section{Recommendations}

Based on the experience of implementing CenterPOINT, the following recommendations can be made for best practices related to development of undergraduate STEM student study spaces.

1. A convenient location near department offices, faculty offices, and classrooms should be selected. Having a study space in the same building as students' classes allows them to utilize the space more frequently, even multiple times per day, before, between, and after classes. More frequent visits are likely to result in stronger connections with other students and increased awareness of campus resources and events. In addition, survey results indicated that location was the number-one factor that influenced students to return to the space.

2. Every attempt should be made to create a comfortable, welcoming space that is inclusive of all students. A comfortable and welcoming space is generally more inviting, which will help to encourage students to visit and return to the center. More frequent visits are likely to result in stronger connections with other students and increased awareness of campus resources and events. In addition, feeling comfortable and welcomed in a space will build students' sense of belonging. Survey results indicated that the atmosphere was the second strongest factor in determining the likelihood of a student returning to the center.

3. Study center spaces should provide access to resources, including technology. Being able to access computers, software, printers, and other resources contributes to the functionality of the location. It makes the space more convenient because it acts as a one-stop-shop for effective study and makes it more likely that students will a) stay longer when they come and b) return more frequently. This is the third biggest factor influencing students to return to the space, according to survey results.

4. It is important for the space to be staffed, and for the staff to have appropriate training and knowledge. Oversight and assessment of the space is essential and is best conducted by a professional staffperson who spends significant time in the space interacting with students. In addition, having a staff that includes student assistants allows for more peer interactions and better connections. Being greeted by a friendly face upon entering the center can contribute positively to the overall atmosphere and inclusive culture of the space, helping users to feel welcome. By having staff who are knowledgeable about the university and its services, the center can help student users make important connections and access campus resources. In survey results, center staff is the fourth most significant factor influencing students to return to the space.

5. If possible, the space should provide access to academic assistance. This does not necessarily mean directly hiring tutors, but may mean partnering with campus offices that already have tutors to suggest holding some hours in the center, or simply providing a space that is 
conducive to tutoring meetings. Having tutors visible in a public space creates a culture where it is acceptable and encouraged to ask for and receive homework and study help. A culture of helping can increase students' confidence in their ability to succeed in difficult STEM courses. Receiving help can also build their skills and knowledge. For the tutors, providing assistance to their peers can build their own confidence, and can also result in positive feelings of pride and belonging within the campus community.

6. It is critical that the center or space be student-focused. This means that students should be included in every step of the planning and implementation process, and that their suggestions should be heard and taken seriously. As part of creating a student-centered space, the set-up should be flexible and allow for multiple learning styles and preferences. In addition, the center hours should be selected to provide access that meets students' preferences, even if it may be outside of normal business hours.

7. The intentional development of collaborative interdepartmental relationships is another important component for the success of the space. It is prudent to involve members of the administration in the center's business early and often and even to include them as part of leadership teams when appropriate. Faculty and instructor support is also necessary. They can encourage students in their classes to take advantage of the resources the space provides, or make referrals for students who may be academically at risk. Consider encouraging faculty to hold a few office hours in the space or provide a short description of the center for them to include in their course syllabi. Communicate that the space can be a resource for them as well as for the students. The use of advisory boards is an effective way to develop partnerships with administrators and faculty and to let others know about the goals and services of the space.

\section{References}

1. Center for the Advancement of Engineering Education. (2010). Enabling engineering student success. (Report No. CAEE-TR-10-02). Retrieved from http://www.engr.washington.edu/caee/

2. Tinto, V. (1987). Leaving college: Rethinking the causes and cures of student attrition. Chicago, IL: University of Chicago Press.

3. U.S. Department of Education, Institute of Education Sciences. (2013). STEM attrition: College students' paths into and out of STEM fields (Report No. NCES 2014-001). Retrieved from http://nces.ed.gov /pubsearch/pubsinfo.asp?pubid=2014001rev

4. U.S. Department of Education, Institute of Education Sciences. (2015). The condition of education (Report No. NCES 2015144). Retrieved from http://nces.ed.gov/pubsearch/pubsinfo.asp?

pubid=2015144 


\section{Appendix A: CenterPOINT Floor Plan Layout}

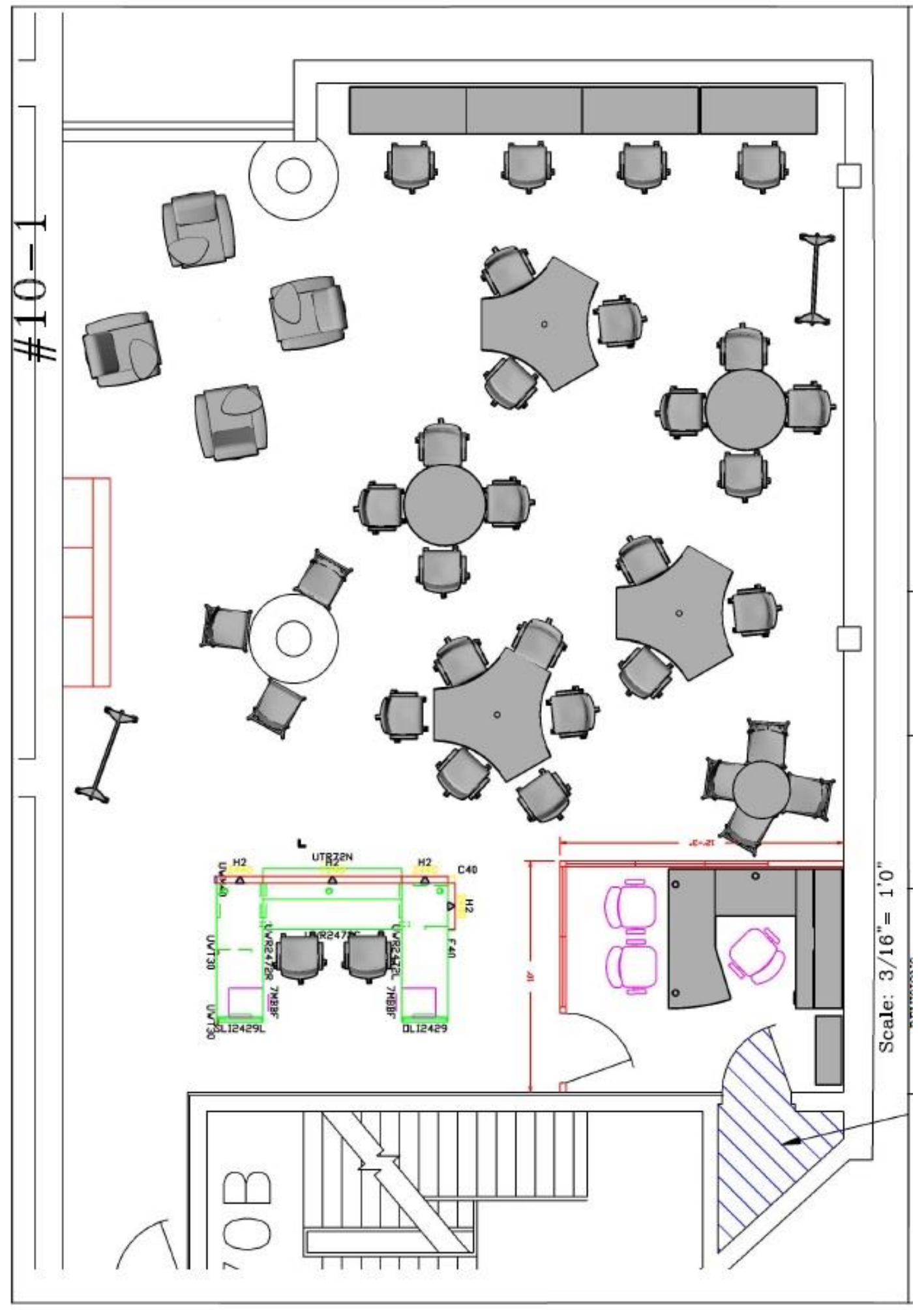




\section{Appendix B: CenterPOINT Annual Survey Questions 2014-15}

1. How often did you visit CenterPOINT during this academic year (Fall 2014 and Spring 2015)?

Almost daily.

Several times per week.

$\square$ About once per week.
A few times.

$\square$ Only once or twice.

2. Which services/resources did you take advantage of? (Check all that apply.)

$\square$ Kitchen

Study tables

$\square$ Lounge chairs

Tutor or PAL

Whiteboards $\square$ Appointment scheduling

Check-out items

Computers

Other; please specify:

3. In what ways did you utilize the CenterPOINT? (Check all that apply.)

$\square$ Individual study

$\square$ Socializing

$\square$ Group study

Other; please specify:

Relaxing

Holding meetings

4. Which factors influenced your decision to visit/return to CenterPOINT? (Check all that apply.)

$\square$ Convenience of location

CenterPOINT staff

$\square$ Tutor or PAL hours

Availability of resources

Atmosphere

Other; please specify:

Kitchen/food

5. Please indicate your level of agreement with the following statements:

a. I feel I fit in at the CenterPOINT.
Strongly Agree
Agree
Disagree
Strongly Disagree

b. CenterPOINT provides a welcoming environment.
Strongly Agree
Agree
Disagree
Strongly Disagree

c. I feel comfortable while at the CenterPOINT.
Strongly Agree
Agree
Disagree
Strongly Disagree

d. If I had a question about campus resources, I feel I could come to the CenterPOINT to ask.

Strongly Agree

Agree

Disagree

Strongly Disagree 
e. CenterPOINT has impacted my academic success.

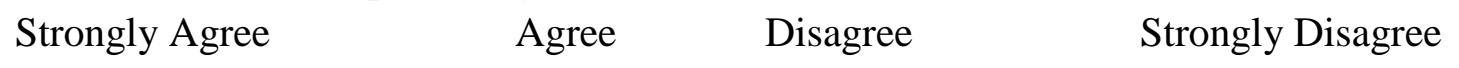

6. How could CenterPOINT better meet your academic needs?

7. In what CenterPOINT-sponsored programs or events did you participate this year? (Check all that apply.)

$\square$ Kimberly-Clark trip

Covance workshops

$\square$ Epic trip

Travel \& Research grants

$\square$ Greenheck Air Tour

$\square$ Dine \& Dish Sessions

8. Please indicate your level of agreement with the following statements:

a. The CenterPOINT staff is welcoming.

Strongly Agree $\quad$ Agree Disagree $\quad$ Strongly Disagree

b. The CenterPOINT staff is knowledgeable.
Strongly Agree
Agree
Disagree
Strongly Disagree

9. Do you have any additional comments?

10. Do you consent to allowing the STEP Grant team to access your academic data, including grade point average and credits earned? (Note: The CenterPOINT and its programs are funded by National Science Foundation STEP Grant \#1161180. This data is requested in order to include in an annual report to NSF. Names will not be used and data will be reported in aggregate so that participating individuals cannot be identified.) 